\title{
CARACTERIZAÇÃO FÍSICA E QUÍMICA DE LIPÍDIOS ESTRUTURADOS OBTIDOS APARTIR DA INTERESTERIFICAÇÃO QUÍMICA DA MISTURA DE MURUMURU E ÓLEO DE BURITI
}

\author{
J. P. P. da SILVA ${ }^{1}$, P. D. de OLIVEIRA ${ }^{1}$, A. M. C. RODRIGUES ${ }^{1}$, L. H. M. da \\ SILVA $^{1}$ \\ ${ }^{1}$ Universidade Federal do Pará, Programa de Pós -Graduação em Ciência e Tecnologia \\ de Alimentos \\ E-mail para contato: jezicapriscila@ hotmail.com
}

\begin{abstract}
RESUMO - A região amazônica apresenta uma grande biodiversidade de espécies vegetais, com potenciais aplicações nas indústrias de alimentos. Os requisitos para a aplicação de lipídios em diversos produtos tem se tornado cada vez mais sofisticado neste contexto a interesterificação tem recebido grande atenção da indústria de modificação de óleos comestíveis como método alternativo para melhorar as propriedades físico-químicas dos óleos e gorduras. Este trabalho teve como objetivo caracterizar os lipídios estruturados obtidos por interesterificação química das misturas de murumuru e óleo de buriti em diferentes proporções $(30: 70 ; 40: 60 ; 50: 50$; 60:40 de murumuru e óleo de buriti, respectivamente). As seguintes análises foram realizadas: perfil de ácidos graxos, acidez, índice de peróxido, índice de iodo, índice de saponificação, ponto de fusão e consistência. O índice de peróxido, a acidez e a consistência das misturas diminuiu após a reação de interesterificação química e o ponto de fusão aumentou. Entre as misturas estudadas, a proporção com $30 \%$ de gordura de murumuru e $70 \%$ de óleo buriti foi a que apresentou os melhores resultados depois da reação de interesterificação química para aplicação em produtos gordurosos apresentando consistência com espalhabilidade satisfatória na temperatura de refrigeração $\left(10^{\circ} \mathrm{C}\right)$, além de conter mais de $55 \%$ de ácidos graxos insaturados, reunindo boas propriedades físicas e nutricionais.
\end{abstract}

\section{INTRODUÇÃO}

Os óleos e gorduras naturais podem ser o único constituinte de um produto ou podem fazer parte da mistura de diversos constituintes em um composto. Existem casos, entretanto, que se torna necessário modificar as características desses materiais, para adequá-los a uma determinada aplicação. (CLAUSS, 1996).

Nesse contexto, a estrutura básica dos óleos e gorduras pode ser redesenhada, por meio da modificação química dos ácidos graxos (hidrogenação), pela reversão da ligação éster (hidrólise) e reorganização dos ácidos graxos na cadeia principal do triglicerídeo (interesterificação) (CASTRO et al., 2004). 
É possível mudar a posição dos radicais de ácidos graxos nos glicerídeos de uma gordura pelo processo conhecido como interesterificação, randomização ou troca de ésteres. Isso é possível porque, na presença de certos catalisadores, os radicais dos ácidos graxos podem se mover entre posições hidroxila, o que resulta em uma distribuição de forma essencialmente randômica (GHOTRA et al., 2002).

O processo de interesterificação permite a modificação no comportamento de óleos e gorduras, oferecendo contribuições importantes para o aumento e otimização do uso dos mesmos nos produtos alimentícios (ERICKSON, 1995).

Visando contribuir para ampliar a qualidade e usos dos óleos vegetais obtidos a partir dos frutos nativos da região amazônica, o objetivo deste trabalho foi caracterizar os lipídios estruturados obtidos por interesterificação química das misturas de murumuru e óleo de buriti.

\section{MATERIAIS E MÉTODOS}

\subsection{Obtenção das Misturas Binárias}

O óleo de buriti e gordura de murumuru brutos foram cedidos pela empresa BERACA, situada em Ananindeua - PA. As matérias primas foram acondicionadas à -10 ${ }^{\circ} \mathrm{C}$, em frascos de $500 \mathrm{~mL}$ até a sua utilização.

As amostras foram preparadas por meio de misturas binárias de gordura de murumuru e óleo de buriti nas proporções (30:70; 40:60; 50:50; 60:40, respectivamente). Em seguida as misturas foram homogeneizadas por 10 minutos e armazenadas sob refrigeração.

\subsection{Interesterificação Química}

A reação de interesterificação química foi realizada sob pressão reduzida, onde $100 \mathrm{~g}$ da mistura foi previamente submetida à secagem em balão de três bocas com agitação magnética, em banho a $80-85^{\circ} \mathrm{C}$ por 40 minutos. A essa mistura foi adicionado $1 \%(\mathrm{~m} / \mathrm{m})$ de catalisador metóxido de sódio.

A reação de interesterificação foi realizada sob agitação magnética constante, pressão reduzida $(20-30 \mathrm{~mm} \mathrm{Hg})$, a $70^{\circ} \mathrm{C}$ por $1 \mathrm{~h}$. Para interromper a reação foi adicionado $5 \mathrm{~mL}$ de água destilada à $60^{\circ} \mathrm{C}$. Finalmente efetuou-se a filtração a quente sob vácuo utilizando-se papel de filtro e sulfato de sódio anidro para a retenção da umidade. Após a filtração a amostra foi novamente submetida á secagem a $80-85^{\circ} \mathrm{C}$, sob pressão reduzida por 40 minutos.

\subsection{Propriedades Físicas e Químicas das Misturas Binárias}


As amostras foram caracterizadas segundo metodologia da AOCS (1997), sendo realizadas as análises: acidez de acordo com método $\mathrm{Ca} 5-40$; índice de iodo pelo método Cd 1- 85; índice de peróxido de acordo com o método $\mathrm{Cd} 8$ - 53; índice de saponificação de acordo com método $\mathrm{Cd} 3^{\mathrm{a}}-94$ e ponto de fusão segundo método Cc 3 -25 .

Composição em ácidos graxos: Os ésteres metílicos das misturas binárias foram preparados pelo método descrito por Rodrigues et al. (2010), sendo a composição em ácidos graxos determinada por cromatografia gasosa, usando gás hélio e vazão de $1 \mathrm{~mL} / \mathrm{min}$.

Consistência: Foi determinada utilizando texturômetro microprocessado e automático, QTS - 25 (BROOKFIELD, USA), operando em interface com um microcomputador, através do software Texture Pro® versão 2.1. As amostras foram acondicionadas por 24 horas à $10^{\circ} \mathrm{C}$, para determinação da consistência.

A análise de consistência foi efetuada por meio de teste de penetração utilizando um probe cônico de acrílico com ponta não truncada e ângulo de $45^{\circ}$ de acordo com D’AGOSTINI et. al., 2000.

Para realizar a conversão dos dados de penetração em um parâmetro independente de peso e do tipo de cone, foi utilizada a equação 1 proposta por Haighton (1959), para o cálculo do "yield value":

$$
C=K x W / p^{1,6}
$$

Onde:

$\mathrm{C}=$ "yield value", em gf/cm2

$\mathrm{K}$ = fator que depende do ângulo do cone (para ângulo de $45^{\circ}$, $\mathrm{K}$ é igual a 4.700)

$\mathrm{W}=$ peso total do sistema, em $\mathrm{g}$ (para penetrômetro de cone)

$\mathrm{P}=$ profundidade de penetração, em $0,1 \mathrm{~mm}$.

\section{RESULTADOS E DISCUSSÃO}

\subsection{Perfil de Ácidos Graxos}

A Tabela 1 mostra a composição em ácidos graxos da gordura de murumuru, do óleo de buriti e das respectivas misturas.

A gordura de murumuru apresentou elevada quantidade de gordura saturada (96,81\%), rica em ácido láurico com $62,76 \%$ e em ácido mirístico com 22,40\%. Enquanto que o óleo de buriti é constituído principalmente por gordura monoinsaturada, destacando-se o ácido oléico $(75,74 \%)$, no entanto, apresenta uma quantidade significativa de gordura saturada $(21,94)$, como ácido palmítico. 
A adição de óleo de buriti à gordura de murumuru provocou uma redução no teor de ácidos graxos saturados. A adição de $40 \%$ de óleo de buriti à gordura provoca uma diminuição de $97 \%$ para cerca de $67 \%$ em ácidos graxos saturados. Por causa da elevada quantidade de gorduras saturada do murumuru, as misturas ainda ficaram com uma elevada quantidade de gordura saturada $(66,87-44,41 \%)$. Desta maneira, o grau de saturação da gordura de murumuru pode ser diminuído pela adição de um óleo vegetal, o que contribuiria não só para melhoras nas características físicas do produto, como também nas propriedades nutricionais (ROUSSEAU et al., 1996).

Tabela 1 - Composição em ácidos graxos da gordura de murumuru, do óleo de buriti e suas misturas binárias

\begin{tabular}{lcccccc}
\hline $\begin{array}{l}\text { Ácidos } \\
\text { Graxos }\end{array}$ & Murumuru & $\begin{array}{c}\text { MB* } \\
(30: 70)\end{array}$ & $\begin{array}{c}\text { MB } \\
(40: 60)\end{array}$ & $\begin{array}{c}\text { MB } \\
(50: 50)\end{array}$ & $\begin{array}{c}\text { MB } \\
(60: 40)\end{array}$ & Buriti \\
\hline C8:0 & 5,46 & 2,05 & 2,54 & 3,03 & 3,51 & 0,59 \\
C10:0 & 1,78 & 0,55 & 0,72 & 0,90 & 1,08 & 0,02 \\
C12:0 & 62,76 & 18,91 & 25,18 & 31,44 & 37,70 & 0,12 \\
C13:0 & 0,08 & 0,02 & 0,03 & 0,04 & 0,05 & - \\
C14:0 & 22,40 & 6,85 & 9,07 & 11,29 & 13,52 & 0,18 \\
C16:0 & 3,32 & 14,86 & 13,22 & 11,57 & 9,92 & 19,81 \\
C16:1 & - & 0,19 & 0,16 & 0,13 & 0,11 & 0,27 \\
C17:0 & - & 0,06 & 0,05 & 0,04 & 0,03 & 0,08 \\
C18:0 & 1,01 & 1,10 & 1,09 & 1,08 & 1,06 & 1,14 \\
C18:1 & 3,19 & 53,98 & 46,72 & 39,46 & 32,21 & 75,74 \\
C18:2 & - & 1,42 & 1,22 & 1,02 & 0,81 & 2,04 \\
$\Sigma$ Saturados & 96,81 & 44,41 & 51,9 & 59,39 & 66,87 & 21,94 \\
$\Sigma$ Monoinst. & 3,19 & 54,17 & 46,88 & 39,59 & 32,32 & 76,1 \\
$\Sigma$ Poliinsat. & - & 1,42 & 1,22 & 1,02 & 0,81 & 2,04 \\
Índice de & 2,87 & 51,26 & 44,35 & 37,43 & 30,52 & 72,02 \\
Iodo & & & & & & \\
\hline
\end{tabular}

Os valores obtidos para o índice de iodo estão em conformidade com o grau de insaturação da gordura de murumuru e óleo de buriti, bem como suas proporções nas misturas.

\subsection{Acidez, Índice de Peróxido e Índice de Saponificação}

A Tabela 2 apresenta os valores de acidez (AGL) e índice de peróxido das misturas antes e após a interesterificação química obtidos experimentalmente, e os valores de saponificação antes da interesterificação.

O óleo de buriti apresentou teor inicial de acidez 3,63\% e a gordura de murumuru 7,83 $\%$. A acidez das misturas foi influenciada pela quantidade de gordura de murumuru, pois quanto maior a proporção desta maior a acidez das misturas. Após a reação de interesterificação, a acidez das misturas teve uma redução significativa. 
Tabela 2 - Acidez, Peróxido e Saponificação das misturas

\begin{tabular}{cccccc}
\hline \multirow{2}{*}{ Misturas } & \multicolumn{2}{c}{$\begin{array}{c}\text { Acidez } \\
\text { (g ácido oléico/100g) }\end{array}$} & \multicolumn{2}{c}{$\begin{array}{c}\text { Peróxido } \\
(\mathrm{meq} / \mathrm{kg})\end{array}$} & $\begin{array}{c}\text { Saponificação } \\
\left(\mathrm{mg} \mathrm{KOH} \mathrm{g}^{-1}\right)\end{array}$ \\
\cline { 2 - 6 } & Antes & Após IQ** & Antes & Após IQ & Antes \\
\hline Buriti & $3,63 \pm 0,01$ & - & $12,32 \pm 0,04$ & - & 194,24 \\
MB* (30:70) & $5,18 \pm 0,06$ & $0,30 \pm 0,01$ & $8,01 \pm 0,01$ & $2,02 \pm 0,05$ & 208.96 \\
MB (40:60) & $5,34 \pm 0,02$ & $0,35 \pm 0,01$ & $5,72 \pm 0,05$ & $2,05 \pm 0,00$ & 214,35 \\
MB (50:50) & $5,73 \pm 0,02$ & $0,37 \pm 0,02$ & $4,51 \pm 0,00$ & $2,86 \pm 0,01$ & 220,04 \\
MB (60:40) & $6,10 \pm 0,02$ & $0,40 \pm 0,00$ & $3,73 \pm 0,04$ & $2,87 \pm 0,01$ & 226,05 \\
Murumuru & $7,83 \pm 0,01$ & - & $0,41 \pm 0,00$ & - & 253,76 \\
\hline
\end{tabular}

*MB: Murumuru e Buriti; **IQ: Interesterificação Química

O índice de peróxido das misturas foi influenciado pela quantidade de óleo de buriti. Após a reação de interesterificação, o índice de peróxido diminuiu para todas as misturas, com valores menores de 2,88 meq/ $\mathrm{kg}$, estando de acordo com a legislação brasileira para óleos e gorduras refinadas que devem apresentar no máximo $10 \mathrm{meq} / \mathrm{kg}$ (ANVISA, 2005).I

$\mathrm{O}$ valor encontrado para o índice de saponificação da gordura de murumuru foi $253,76 \mathrm{mg} \mathrm{KOH} \mathrm{g}^{-1}$ e para o óleo de buriti foi de 194,24 $\mathrm{mg} \mathrm{KOH} \mathrm{g}^{-1}$. As misturas binárias obtiveram valores que variaram de 208,96 - 226,05 $\mathrm{mg} \mathrm{KOH} \mathrm{g}^{-1}$, sendo influenciadas pela quantidade de gordura de murumuru, quanto maior a proporção de gordura maior índice de saponificação.

\subsection{Ponto de Fusão}

A Tabela 3 apresenta os valores de ponto de fusão das misturas obtidos experimentalmente antes e após a interesterificação química.

Tabela 3 - Ponto de fusão das misturas antes e após a interesterificação química

\begin{tabular}{ccc}
\hline Misturas & \multicolumn{2}{c}{ Ponto de fusão $\left({ }^{\circ} \mathrm{C}\right)$} \\
MB* & Antes IQ** & Depois IQ \\
\hline MB (30:70) & $24,2 \pm 0,10$ & $24,6 \pm 0,17$ \\
MB (40:60) & $26,1 \pm 0,12$ & $26,8 \pm 0,10$ \\
MB (50:50) & $27,0 \pm 0,06$ & $28,6 \pm 0,06$ \\
MB (60:40) & $28,1 \pm 0,10$ & $28,9 \pm 0,06$ \\
\hline
\end{tabular}

*MB: Murumuru e Buriti; **IQ: Interesterificação Química

De acordo com a Tabela 3, a adição do óleo de buriti à gordura de murumuru provocou uma diminuição no ponto de fusão das misturas, antes e após a interesterificação química. Enquanto que a interesterificação provocou um aumento do ponto de fusão das misturas binárias, isso porque, o processo de interesterificação permite alterar a distribuição dos ácidos graxos nos triacilgliceróis, formando produtos 
com características de fusão e cristalização diferentes dos óleos e gorduras originais (CHIU e GIOIELLI, 2008).

Azevedo-Meleiro e Gonçalves (2005) avaliaram algumas propriedades de 28 gorduras técnicas comerciais das indústrias brasileira, verificaram que os pontos de fusão dessas gorduras variavam de 16,6 a $47,3^{\circ} \mathrm{C}$. Suas aplicações são variadas, sendo utilizadas em chocolates $\left(39^{\circ} \mathrm{C}\right)$, sovertes $\left(24,9^{\circ} \mathrm{C}\right)$, panificação $\left(16,6^{\circ} \mathrm{C}\right)$, dentre outros. Portanto, com relação à aplicação das misturas estudadas, a mistura com proporção 30:70 de murumuru/buriti após a interesterificação química apresentou ponto de fusão adequado à elaboração de sorvete $\left(24,6^{\circ} \mathrm{C}\right)$.

\subsection{Consistência}

A Tabela 4 apresenta a consistência das misturas antes e pós a interesterificação química, a $10^{\circ} \mathrm{C}$.

Tabela 4 - Consistência a $10^{\circ} \mathrm{C}$ da mistura de murumuru e óleo de buriti antes e após a interesterificação química

\begin{tabular}{ccc}
\hline \multirow{2}{*}{ MB* $^{*}$} & \multicolumn{2}{c}{ Consistência $\left(\mathrm{gf} / \mathrm{cm}^{2}\right)$} \\
MB (30:70) & $1567,93 \pm 90,13$ & Após IQ \\
\hline MB (40:60) & $3182,73 \pm 151,82$ & $988,84 \pm 29,94$ \\
MB (50:50) & $4859,52 \pm 303,63$ & $2183,30 \pm 149,68$ \\
MB (60:40) & $6140,17 \pm 186,03$ & $2978,61 \pm 239,49$ \\
\hline
\end{tabular}

*MB: Murumuru e Buriti; **IQ: Interesterificação Química

A adição de óleo de buriti a gordura de murumuru provocou uma redução na consistência das misturas. A interesterificação química provocou uma nítida diminuição na consistência de todas as misturas, a temperatura analisada $d e 10^{\circ} \mathrm{C}$, a consistência reduziu de 16 a $39 \%$.

As gorduras podem ser classificadas quanto à sua espalhabilidade em função de seu "yield value" ou consistência, de acordo com os critérios estabelecidos por Haighton (1959). Uma gordura pode ser considerada espalhável na faixa entre 100 e $1000 \mathrm{gf} / \mathrm{cm}^{2}$.

Os resultados demonstraram que, à $10^{\circ} \mathrm{C}$, todas as misturas antes e após a interesterificação química apresentaram consistência muito dura mesmo com a redução na consistência provocada pela reação. Exceto, a mistura 30\% de murumuru e $70 \%$ de óleo de buriti após a interesterificação, que apresentou consistência dura, mas satisfatoriamente espalhável $\left(988,84 \mathrm{gf} / \mathrm{cm}^{2}\right)$. Rodrigues et al. (2004) verificaram a consistência de creme vegetal com fitosteróis que apresentou valor de $930,8 \mathrm{gf} / \mathrm{cm}^{2}$, valor próximo ao encontrado nesse estudo após a interesterificação para amostra 30:70, o que significa espalhabilidade satisfatória à temperatura de refrigeração para ambos. 


\section{CONCLUSÃO}

Entre as misturas estudadas, a proporção de 30:70 (murumuru/buriti) foi a que apresentou os melhores resultados depois da reação de interesterificação química para aplicação em produtos gordurosos apresentando consistência com espalhabilidade satisfatória na temperatura de refrigeração $\left(10^{\circ} \mathrm{C}\right)$, bom ponto de fusão, além de conter mais de $55 \%$ de ácidos graxos insaturados, reunindo boas propriedades físicas e nutricionais.

\section{REFERÊNCIAS BIBLIOGRÁFICAS}

ANVISA, AGENCIA DE VIGILÂNCIA SANITÁRIA. Legislação. Resolução n. 270 de 22 de setembro de 2005. Aprova regulamento técnico sobre óleos vegetais, gorduras vegetais e creme vegetal. Dispinível em $<$ http://portal.anvisa.gov.br/wps/wcm/connect>. Acesso em: 25 de dezembro de 2013.

AOCS, AMERICAN OIL CHEMISTS' SOCIETY. Official methods and recommended practices of the AOCS. $4^{\text {a }}$.ed. Champaing, 1997.

AZEVEDO-MELEIRO, C.H. de; GONÇALVES, L.A.G. Teores de ácidos graxos trans em gorduras hidrogenadas comerciais brasileiras. Revista Universidade Rural: Série Ciências Exatas e da Terra, Seropédica, RJ: EDUR, v. 24, n. 1-2, p. 75-81, jan-dez., 2005.

CASTRO, H.F.; MENDES, A.A.; SANTOS J.C.; AGUIAR, C.L.; Modificação de óleos e gorduras por biotransformação. Química Nova vol.27 no.1 São Paulo Jan./Feb. 2004.

CHIU, M.C.; GIOIELLI, L.A. Lipídios estruturados obtidos a partir da mistura de gordura de frango, sua estearina e triacilgliceróis de cadeia média. ii- pontos de amolecimento e fusão. Quimica Nova, vol. 31, n 2, 238-243, 2008.

CLAUSS, J.; Óleos \& Grãos 1996, 5, 31.

D’AGOSTINI, D.; FERRAZ, R.C.; GIOIELLI, L.A. Consistência de misturas binárias e ternárias de gorduras de palma, palmiste e triacilgliceróis de cadeia média. Revista Brasileira Ciências Farmacêuticas, São Paulo, v.36, nº1, p.147-155, 2000 .

ERICKSON, D. R.; Practical Handbook of Soybean Processing and Utilization, AOCS Press: Champaign, Illinois, 1995.

GHOTRA, B.S.; DYAL, S.D.; NARINE, S.S. Lipid shortenings: a review. Food Research International. 35, 1015-1048, 2002.

HAIGHTON, A.J. The measurement of the hardness of margarine and fat with con penetrometers. Journal of the American Oil Chemists Society, Chicago, v. 36, n. 8, p. 345-348, 1959. 
RIBEIRO, A.P.B; MOURA, J. M. L. N.; GRIMALDI, R.; GONÇALVES, L.A.G.. Interesterificação química: alternativa para obtenção de gorduras zero trans. Quim. Nova, Vol. 30, No. 5, 1295-1300, 2007.

RODRIGUES, A.M.C.; DARNET, S.; SILVA, L.H.M. Fatty Acid Profiles and Tocopherol Contents of Buriti (Mauritia flexuosa), Patawa (Oenocarpus bataua), Tucuma (Astrocaryum vulgare), Mari (Poraqueiba paraensis) and Inaja (Maximiliana maripa) Fruits. Journal Brazilian Chem. Society. v.21, No. 10, 2000-2004, 2010.

RODRIGUES, J. N.; MANCINI FILHO, J.; TORRES, R. P.; GIOIELLI, L. A.. Caracterização físico-química de creme vegetal enriquecido com ésteres de fitosteróis. Rev. Bras. Cienc. Farm. Braz. J. Pharm. Sci. vol. 40, n. 4, out./dez., 2004.

ROUSSEAU, D., FORESTIERE, K., HILL, A. R., MARANGONI, A. G. Restructuring butterfat through blending and chemical interesterification. 1. Melting behavior and triacylglycerol modifications. Journal of the American Oil Chemists. Society, Champaign, v. 73, n. 8, p. 963- 972, 1996. 